\title{
Current Standard and Future Perspectives in First- and Second-Line Treatment of Metastatic Pancreatic Adenocarcinoma
}

\author{
Volker Ellenrieder ${ }^{\mathrm{a}} \quad$ Alexander König $^{\mathrm{a}}$ Thomas Seufferlein $^{\mathrm{b}}$ \\ a Department of Gastroenterology and Gastrointestinal Oncology, University Medical Center Göttingen, Göttingen, and \\ ${ }^{\mathrm{b}}$ Department of Internal Medicine I, University of UIm, Ulm, Germany
}

\section{Key Words}

Pancreatic cancer · Chemotherapy · Palliation

\begin{abstract}
Background: Pancreatic ductal adenocarcinoma (PDAC) is a highly aggressive malignancy with a median 5-year survival of $<8 \%$. At the time of diagnosis, a vast majority of pancreatic cancer patients were found to be with either metastatic spread of the disease or locally advanced tumors. Despite relatively low efficacy, gemcitabine administration was the first choice chemotherapeutic strategy in advanced PDAC for many years. In the last 5 years, however, our understanding of pancreatic carcinogenesis has improved dramatically and with this our therapeutic options have expanded significantly. Summary: With the FOLFIRINOX protocol or the combination of gemcitabine and nab-paclitaxel, 2 novel and more effective chemotherapeutic regimens have been introduced in clinical routine, which increased the overall survival by $4-5$ months in the palliative situation. Most recently, we learned that both regimens can be modified and dosages can be adapted in older patients without significant loss of efficacy. Additionally, novel application strategies such as nanoparticle fused liposomal irinotecan along with 5-FU/LV provided convincing results in patients previously treated with gemcitabine. Current preclinical and clinical trials investigate efficacy and tolerability of novel drugs aiming at the
\end{abstract}

inhibition of key inflammatory pathways, for example, JAKSTAT signaling, or the tumor surrounding desmoplasia. Prospectively, immunovaccination approaches or immune checkpoint inhibition appears as promising strategies in the near future, particularly when combined with epigenetic drugs in advanced PDAC patients. In this 'to-the-point' article, we review the current standard and summarize the most recent and encouraging advances in cytostatic PDAC treatment. Key Points: (1) FOLFIRINOX and nab-paclitaxel/gemcitabine as first-line treatment regime significantly increase survival in patients with advanced PDAC; (2) Selection of appropriate treatment regime depends on patient performance, comorbidity, and toxicity; (3) PDAC patients will benefit from second-line chemotherapy and selection of appropriate regimes depends on first line therapy and patient criteria; (4) Future therapeutic strategies in advanced PDAC will respect molecular tumor profiling and other biomarkers.

(c) 2016 S. Karger AG, Basel

\section{Established First-Line Treatment Concepts in Metastatic PDAC}

For almost 15 years, cytostatic monotherapy with gemcitabine was used as standard treatment for metastatic pancreatic cancer. In 1997, Burris et al. [1] have shown that gemcitabine efficacy is superior to 5-FU with

\section{KARGER}

E-Mail karger@karger.com

www.karger.com/dig (c) 2016 S. Karger AG, Basel

$0012-2823 / 16 / 0941-0044 \$ 39.50 / 0$
Prof. Dr. Volker Ellenrieder

Department of Gastroenterology and Gastrointestinal Oncology

University Medical Center Göttingen

Robert Koch Strasse 40, DE-37075 Göttingen (Germany)

E-Mail volker.ellenrieder@ med.uni-goettingen.de 
Table 1. Clinical trials in first line therapy of metastatic pancreatic cancer

\begin{tabular}{|c|c|c|c|c|c|c|c|c|c|}
\hline Trial name & $\begin{array}{l}\text { Trial } \\
\text { phase/arms }\end{array}$ & $\begin{array}{l}\text { Year } \\
\text { published }\end{array}$ & $\begin{array}{l}\text { Number of } \\
\text { patients } \\
\text { enrolled }\end{array}$ & $\begin{array}{l}\text { Incestigated } \\
\text { drugs }\end{array}$ & $\begin{array}{l}\text { Clinical } \\
\text { outcome (OS) }\end{array}$ & $\mathrm{p}$ value & $\begin{array}{l}\text { Clinical } \\
\text { outcome } \\
\text { (ORR) }\end{array}$ & $\mathrm{p}$ value & First author \\
\hline & $\begin{array}{l}\text { Phase II } \\
2 \text { arms }\end{array}$ & 1997 & $\begin{array}{l}126 \text { patients } \\
\text { (1:1 ratio) }\end{array}$ & $\begin{array}{l}\text { Gemcitabine } \\
\text { vs. } 5 \text {-FU }\end{array}$ & $\begin{array}{l}5.65 \text { vs. } \\
4.4 \text { month }\end{array}$ & 0.0025 & $\begin{array}{l}5.4 \text { vs. } \\
0 \%\end{array}$ & & $\begin{array}{l}\text { Burris } \\
\text { et al. [1] }\end{array}$ \\
\hline NCIC CTG PA. 3 & $\begin{array}{l}\text { Phase III } \\
2 \text { arms }\end{array}$ & 2007 & $\begin{array}{l}569 \text { patients } \\
(1: 1 \text { ratio })\end{array}$ & $\begin{array}{l}\text { Gemcitabine vs. } \\
\text { gemcitabine + } \\
\text { erlotinib }\end{array}$ & $\begin{array}{l}5.91 \text { vs. } \\
6.24 \text { month } \\
10.51 \text { month }^{\#}\end{array}$ & $\begin{array}{c}0.038 \\
<0.0001\end{array}$ & $\begin{array}{l}8.0 \text { vs. } \\
8.6 \%\end{array}$ & & $\begin{array}{l}\text { Moore } \\
\text { et al. [10] }\end{array}$ \\
\hline $\begin{array}{l}\text { Prodige } 4- \\
\text { ACCORD } 11\end{array}$ & $\begin{array}{l}\text { Phase II/III } \\
2 \text { arms }\end{array}$ & 2011 & $\begin{array}{l}342 \text { patients } \\
(1: 1 \text { ratio) }\end{array}$ & $\begin{array}{l}\text { Gemcitabine vs. } \\
\text { oxaliplatin }+ \\
\text { irinotecan }+ \\
\text { leucovorin }+5-\mathrm{FU}\end{array}$ & $\begin{array}{l}6.8 \text { vs. } \\
11.1 \text { month }\end{array}$ & $<0.0001$ & $\begin{array}{l}9.4 \mathrm{vs} . \\
31.6 \%\end{array}$ & 0.0001 & $\begin{array}{l}\text { Conroy } \\
\text { et al. [12] }\end{array}$ \\
\hline MPACT & $\begin{array}{l}\text { Phase III } \\
2 \text { arms }\end{array}$ & 2013 & $\begin{array}{l}861 \text { patients } \\
(1: 1 \text { ratio })\end{array}$ & $\begin{array}{l}\text { Gemcitabine vs. } \\
\text { gemcitabine }+ \\
\text { nab-paclitaxel }\end{array}$ & $\begin{array}{l}6.7 \text { vs. } \\
8.5 \text { month }\end{array}$ & $<0.00015$ & $\begin{array}{l}7 \text { vs. } \\
23 \%\end{array}$ & 0.0001 & $\begin{array}{l}\text { Von Hoff } \\
\text { et al. [14] }\end{array}$ \\
\hline
\end{tabular}

\# Patients showing grad 2 skin toxicity.

respect to overall survival (5.6 vs. 4.4 months), performance status and pain control (table 1). Moreover, gemcitabine treatment is well tolerated and severe side effects, such as hematotoxicity, vomiting, and increased liver enzymes, are found only in a small number of patients [2]. Since then, a large series of clinical trials was conducted to test whether gemcitabine responsiveness can be increased when combined with chemotherapeutic drugs (e.g. capecitabine, oxaliplatin, irinotecan and cisplatin), small molecule inhibitors or monoclonal antibodies against receptor tyrosine kinases or oncogenic growth factors. Unfortunately, despite promising results in preclinical approaches, none of these combinations were able to significantly prolong progression free or overall survival when compared with gemcitabine monotherapy [2-6].

None of the monoclonal antibodies (e.g. bevacizumab, cetuximab, ganitumab) were able to improve gemcitabine-induced survival when tested in phase III studies (e.g. CALGB, SWOG, and GAMMA trials), and similarly, administration of small molecule inhibitors (e.g. sorafenib, tipifarnib, rigosertib, trametinib, aflibercept, sunitinib, everolimus and vismodegib) failed to increase the efficacy of gemcitabine in terms of disease progression and overall survival [2, 7-9]. A sole exception from the disappointing use of small molecule inhibitors was demonstrated in 2007 by Moore et al. [10]. In fact, a modest but significantly improved median overall survival (6.24 vs. 5.91 months) and PFS (3.75 vs. 3.55 months) were shown for gemcitabine when applied in combina- tion with erlotinib, a small molecule inhibitor of the epidermal growth factor receptor (table 1). Moreover, detailed patients analysis identified a subgroup of patients developing a characteristic skin rash and this cohort responded remarkably to the treatment with erlotinib. In fact, a combination of gemcitabine with erlotinib prolonged survival in those patients up to 10.5 months. Although mechanistic proof for this correlation could not be found, it is now accepted that development and severity of skin rash may serve as a biomarker for responsiveness to erlotinib treatment in advanced Pancreatic ductal adenocarcinoma (PDAC) patients. Of note, since higher doses of erlotinib can worsen the severity of skin irritations without improving clinical benefit, dose escalation cannot be recommended [11].

Together, despite much effort, an ideal partner for gemcitabine-based therapy was not found and therefore, gemcitabine monotherapy remained the standard of care for patients with advanced pancreatic cancer. However, the concept of palliative pancreatic cancer treatment changed fundamentally following the publication of the landmark PRODIGE4-ACCORD11 trial in 2011 (table 1). In this randomized, multicenter phase II/III study, the FOLFIRINOX regimen consisting of a combination of 4 intravenously administered drugs (oxaliplatin, leucovorin (calcium folinate), irinotecan and 5-FU) significantly improved patient survival compared to standard singleagent gemcitabine. In total, 336 untreated patients with metastatic PDAC and good performance status (ECOG $0-1$ ) were enrolled and treated with either FOLFIRINOX 


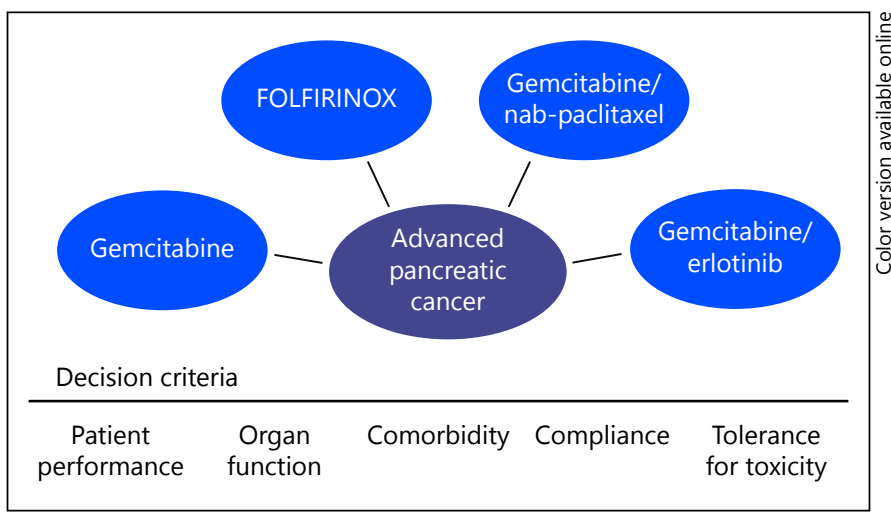

Fig. 1. Chemotherapeutic options for first-line treatment in patients with advanced PDAC and criteria that influence decision for the appropriate treatment regime.

or gemcitabine. The median overall survival was 11.1 months in the FOLFIRINOX arm vs. 6.8 months in the gemcitabine arm; the median progression-free survival was 6.4 vs. 3.3 months and the objective response rate was 31.6 vs. $9.4 \%$, respectively [12]. However, better survival rates and clinical responses came along with the expense of excess toxicity, in particular febrile neutropenia, thrombocytopenia, peripheral neuropathy, fatigue, vomiting and diarrhea, which frequently caused treatment termination. Of note, despite higher frequency of side effects, patients receiving FOLFIRINOX had less deterioration in quality of life compared to patients who had undergone gemcitabine treatment: 31 vs. $66 \%$, respectively [13].

Another new and promising strategy in PDAC treatment targets the tumor microenvironment for enrichment of cytostatic drug concentrations. In the MPACT trial, cytostatic paclitaxel covalently bound to albumin nano-particles was administered in combination with gemcitabine to patients with advanced PDAC [14]. In this study, 861 untreated metastatic PDAC patients were treated either with nab-paclitaxel plus gemcitabine or gemcitabine alone. Combined treatment resulted in a significant increase in median overall survival of 8.5 vs. 6.7 months, respectively, and long-term survival (4\%) has been reported in the nap-paclitaxel/gemcitabine group only (table 1 ). The 1-year survival rate (35 vs. $22 \%$ ), the progression free survival (5.5 vs. 3.7 months) and the objective response rate ( 23 vs. $7 \%$ ) were higher in the nab-paclitaxel plus gemcitabine arm compared to gemcitabine $[14,15]$. Toxicity, mostly grades $3-4$ neutropenia, fatigue and neuropathy, was moderate and toxicity-related deaths were similar (about $4 \%$ ) in both groups. Additional subgroup analysis revealed that patients with lower performance status and a more bulky disease with liver metastasis and $>3$ metastatic sites benefitted most from nab-paclitaxel plus gemcitabine treatment.

\section{Choice for Metastatic Pancreatic Cancer: FOLFIRINOX or Gemcitabine/Nab-Paclitaxel?}

A large cohort analysis has recently confirmed the broad use of the new combination regimens in PDAC and hence, demonstrated that a majority of patients with metastatic diseases are currently treated with either nab-paclitaxel $(43 \%)$ or FOLFIRINOX (14\%), while only $21 \%$ are selected for gemcitabine monotherapy. Currently, one of the most intriguing challenges in metastatic pancreatic cancer treatment is to define selection criteria between the new standard options (fig. 1). Unfortunately, head-to-head studies comparing the new standard regimens are still missing, but available data from clinical trials and experience from daily clinical practice suggest that treatment decision should be made with respect to performance status, patient preference of treatment routes and toxicity profiles (fig. 1). FOLFIRINOX might have higher efficacy and seems to be appropriate in patients with good performance status, while gemcitabine plus nab-paclitaxel treatment can be used in a broader group of patients including those with KPS 70-80 and elderly patients. Importantly, there is now first-hand evidence suggesting that a modest dose reduction of 5-FU and irinotecan by $25 \%$ in the FOLFIRINOX (mFOLFIRINOX) regime can significantly improve the side-effect profile (e.g. fatigue and neutropenia) without compromising efficacy [16]. These findings, however, need to be confirmed in larger series before general recommendations can be given and there are many other modifications of this protocol in clinical use.

\section{Second- and Third-Line Treatment Strategies in PDAC}

Overall, for pancreatic cancer patients whose disease progresses on first-line treatment, the median survival thereafter is about 3-4 months without further tumorspecific treatment. Recently, it could be demonstrated that patients benefit from a second-line treatment. The issue is which protocols are the most appropriate in terms of efficacy and tolerability (fig. 2). In the last 5 years, var- 


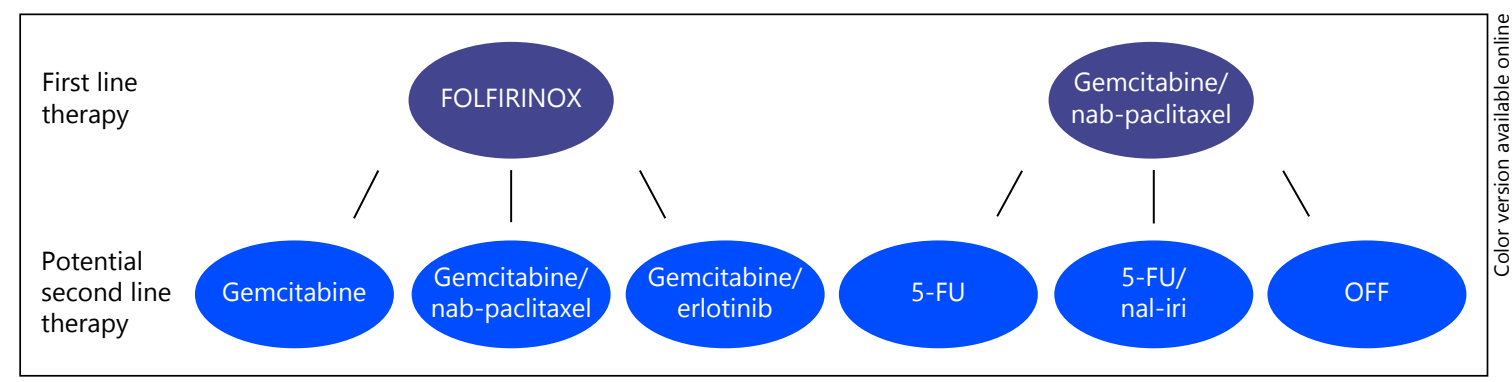

Fig. 2. Chemotherapeutic regimes for second-line treatment in advanced PDAC according to first-line therapy.

ious chemotherapeutic drugs and protocols were studied. Until today, there is no clear standard treatment strategy for second-line therapy in advanced pancreatic cancer. However, this also depends on the first-line treatment (fig. 2). It can also be difficult to administer second-line therapy in pancreatic cancer as a patient's performance status often declines rapidly when the tumor is locally or systemically progressing. This has created real challenges when conducting clinical trials in this setting. Only recently, there has been increasing interest also by pharmaceutical companies to evaluate novel protocols in this setting. Consequently, there are few phase II and III studies demonstrating a benefit from second-line chemotherapy in patients pretreated with a gemcitabine-based regimen. The CONKO 003 phase II trial demonstrated a significant increase in patient survival when treated with 5-FU/LV plus oxaliplatin (OFF) (4.8 vs. 2.3 months) compared to best supportive care [17]. In a subsequent study, the OFF protocol was shown superior to treatment with 5-fluorouracil (5-FU), particularly with respect to overall survival (5.9 vs. 3.3 months) [18]. An alternative regimen, which was reported by the AIO study group (PK0160) showed a progression-free survival of 7 weeks and overall survival of 40 weeks in patients treated with oxaliplatin and docetaxel [19].

The NAPOLI-1 trial most recently presented secondline efficacy data for nanoparticle-bound liposomal irinotecan (nal-iri) when administered in combination with 5-FU/LV [20]. It has been shown that nanoparticle-bound irinotecan significantly accumulates at the tumor site, and this might be associated with higher anti-tumor activity compared to conventional irinotecan. The NAPOLI-1 trial included 417 patients that were pretreated with gemcitabine. The primary endpoint was overall survival. Application of nal-iri alone failed to provide any additional survival benefit over standard therapy. However, in combination with $5-\mathrm{FU} / \mathrm{LV}$, nal-iri significantly increased overall survival (6.1 vs. 4.2 months). Progres- sion-free survival also improved significantly, from 1.5 months with the standard therapy to 3.1 months in patients receiving nal-iri plus 5-FU/leucovorin. Combination therapy was associated with increased gastrointestinal side effects, particularly diarrhea, vomiting and fatigue. In October 2015, the nal-iri plus 5-FU/leucovorin combination was approved by the US Food and Drug Administration for the treatment of patients with metastatic adenocarcinoma of the pancreas after disease progression following gemcitabine-based therapy.

Due to better treatment success in first-line settings, second-line approaches are becoming increasingly more relevant in the medical treatment of pancreatic cancer. Regimen decision should be made based on patient performance status and the selection of cytostatic drugs in the first-line approach. The combination of nanoliposomal irinotecan with 5-FU and leucovorin might be an appropriate choice for patients pretreated with gemcitabine plus nab-paclitaxel. It is not clear whether the sequential use of FOLFIRINOX or nab-paclitaxel is beneficial for patients pretreated with gemcitabine or merely increases chemotherapy related side effects, although first promising results have been reported lately. In fact, Portal et al. [21] presented interesting data from a retrospective analysis of patients who received at least 1 pretreatment protocol with either gemcitabine or 5-FU/LV containing protocols. Interestingly, prior administration of gemcitabine had no effect on treatment efficacy of nab-paclitaxel plus gemcitabine in second- or third-line approaches. Moreover, recent data from non-randomized cohort studies suggest that administration of nab-paclitaxel and gemcitabine in patients' refractory to the FOLFIRINOX regime, might be a successful strategy to delay tumor progression, as documented by an objective response rate of $17.5 \%$. However, the exciting results came along with a high rate of toxicity. Grades 3-4 toxicity occurred in $40 \%$ of the patients, mostly neutropenia and neurotoxicity. Together, increased efficacy and better patient manage- 
ment in first-line approaches allow consideration of second and even third-line treatment options in pancreatic cancer patients with advanced diseases.

\section{Perspectives}

Despite encouraging advances in first- and second-line treatment, pancreatic cancer remains one of the greatest challenges in medical oncology. In fact, there is an urgent need to better select patients for current therapies and develop novel and more efficient therapeutic strategies. Due to recent whole genome sequencing analysis, our understanding of the mutational landscape in pancreatic cancer has increased tremendously. From these recent studies, we learned that the genetic make-up of the disease is highly complex and heterogeneous and reflected by numerous molecular subtypes. Although the molecular classification of pancreatic cancer is still incomplete, there is now emerging consensus suggesting that molecular tumor stratification is key not only for better understanding of the phenotypic subtype behavior but also for predicting therapeutic responsiveness to agents targeting specific pathways or genomic features [22, 23]. In addition, novel approaches targeting key epigenetic events (e.g. HDAC inhibition) or the tumor environment (e.g. the stromal compartment or local immune cell responses) are promising in subsets of pancreatic cancer patients.
Therefore, the next generation of clinical trials must be designed to select treatment strategies for each study patient based on results from molecular stratification of its tumor.

\section{Key Points}

(1)FOLFIRINOX and nab-paclitaxel/gemcitabine as first-line treatment regime significantly increase survival in patients with advanced PDAC;

(2) Selection of appropriate treatment regime depends on patient performance, comorbidity, and toxicity;

(3) PDAC patients will benefit from second-line chemotherapy and selection of appropriate regimes depends on first line therapy and patient criteria;

(4) Future therapeutic strategies in advanced PDAC will respect molecular tumor profiling and other biomarkers.

\section{Acknowledgments}

We would like to thank Dr. U. Koenig for critical revision of the manuscript.

\section{Disclosure Statement}

None.

\section{References}

$\checkmark 1$ Burris HA 3rd, Moore MJ, Andersen J, Green MR, Rothenberg ML, Modiano MR, et al: Improvements in survival and clinical benefit with gemcitabine as first-line therapy for patients with advanced pancreas cancer: a randomized trial. J Clin Oncol 1997;15:24032413.

$\checkmark 2$ Arslan C, Yalcin S: Current and future systemic treatment options in metastatic pancreatic cancer. J Gastrointest Oncol 2014;5:280 295.

3 Berlin JD, Catalano P, Thomas JP, Kugler JW, Haller DG, Benson AB 3rd: Phase III study of gemcitabine in combination with fluorouracil versus gemcitabine alone in patients with advanced pancreatic carcinoma: eastern cooperative oncology group trial E2297. J Clin Oncol 2002;20:3270-3275.

-4 Cunningham D, Chau I, Stocken DD, Valle JW, Smith D, Steward W, et al: Phase III randomized comparison of gemcitabine versus gemcitabine plus capecitabine in patients with advanced pancreatic cancer. J Clin Oncol 2009;27:5513-5518.
5 Hidalgo M: Pancreatic cancer. N Engl J Med 2010;362:1605-1617.

$\checkmark 6$ Heinemann V, Vehling-Kaiser U, Waldschmidt D, Kettner E, Märten A, Winkelmann $\mathrm{C}$, et al: Gemcitabine plus erlotinib followed by capecitabine versus capecitabine plus erlotinib followed by gemcitabine in advanced pancreatic cancer: final results of a randomised phase 3 trial of the 'arbeitsgemeinschaft internistische onkologie' (AIO-PK0104). Gut 2013;62: 751-759.

7 Catenacci DV, Junttila MR, Karrison T, Bahary N, Horiba MN, Nattam SR, et al: Randomized phase Ib/II study of gemcitabine plus placebo or vismodegib, a hedgehog pathway inhibitor, in patients with metastatic pancreatic cancer. J Clin Oncol 2015;33:4284-4292.

-8 Gonçalves A, Gilabert M, François E, Dahan L, Perrier H, Lamy R, et al: BAYPAN study: a double-blind phase III randomized trial comparing gemcitabine plus sorafenib and gemcitabine plus placebo in patients with advanced pancreatic cancer. Ann Oncol 2012; 23:2799-2805.
9 Iovanna J, Mallmann MC, Gonçalvès A, Turrini O, Dagorn JC: Current knowledge on pancreatic cancer. Front Oncol 2012;2:6.

-10 Moore MJ, Goldstein D, Hamm J, Figer A, Hecht JR, Gallinger S, et al; National Cancer Institute of Canada Clinical Trials Group: Erlotinib plus gemcitabine compared with gemcitabine alone in patients with advanced pancreatic cancer: a phase III trial of the national cancer institute of canada clinical trials group. J Clin Oncol 2007;25:19601966.

11 Van Cutsem E, Li CP, Nowara E, Aprile G, Moore M, Federowicz I, et al: Dose escalation to rash for erlotinib plus gemcitabine for metastatic pancreatic cancer: the phase II RACHEL study. Br J Cancer 2014;111:20672075.

12 Conroy T, Desseigne F, Ychou M, Bouché O, Guimbaud R, Bécouarn Y, et al; Groupe Tumeurs Digestives of Unicancer; PRODIGE Intergroup: FOLFIRINOX versus gemcitabine for metastatic pancreatic cancer. N Engl J Med 2011;364:1817-1825. 
13 Gourgou-Bourgade S, Bascoul-Mollevi C, Desseigne F, Ychou M, Bouché O, Guimbaud $\mathrm{R}$, et al: Impact of FOLFIRINOX compared with gemcitabine on quality of life in patients with metastatic pancreatic cancer: results from the PRODIGE 4/ACCORD 11 randomized trial. J Clin Oncol 2013;31:23-29.

14 Von Hoff DD, Ervin T, Arena FP, Chiorean EG, Infante J, Moore M, et al: Increased survival in pancreatic cancer with nab-paclitaxel plus gemcitabine. N Engl J Med 2013;369: 1691-1703.

15 Goldstein D, El-Maraghi RH, Hammel P, Heinemann V, Kunzmann V, Sastre J, et al: Nab-paclitaxel plus gemcitabine for metastatic pancreatic cancer: long-term survival from a phase III trial. J Natl Cancer Inst 2015;pii: dju413.

16 Gunturu KS, Yao X, Cong X, Thumar JR, Hochster HS, Stein SM, et al: FOLFIRINOX for locally advanced and metastatic pancreatic cancer: single institution retrospective review of efficacy and toxicity. Med Oncol 2013; 30:361.
17 Pelzer U, Schwaner I, Stieler J, Adler M, Seraphin J, Dörken B, et al: Best supportive care (BSC) versus oxaliplatin, folinic acid and 5 -fluorouracil (OFF) plus BSC in patients for second-line advanced pancreatic cancer: a phase III-study from the German CONKOstudy group. Eur J Cancer 2011;47:16761681.

18 Oettle H, Riess H, Stieler JM, Heil G, Schwaner I, Seraphin J, et al: Second-line oxaliplatin, folinic acid, and fluorouracil versus folinic acid and fluorouracil alone for gemcitabine-refractory pancreatic cancer: outcomes from the CONKO-003 trial. J Clin Oncol 2014;32: 2423-2429.

19 Ettrich TJ, Perkhofer L, von Wichert G, Gress TM, Michl P, Hebart HF, et al: DocOx (AIO-PK0106): a phase II trial of docetaxel and oxaliplatin as a second line systemic therapy in patients with advanced pancreatic ductal adenocarcinoma. BMC Cancer 2016; 16:21.
20 Wang-Gillam A, Li CP, Bodoky G, Dean A, Shan YS, Jameson G, et al; NAPOLI-1 Study Group: Nanoliposomal irinotecan with fluorouracil and folinic acid in metastatic pancreatic cancer after previous gemcitabine-based therapy (NAPOLI-1): a global, randomised, open-label, phase 3 trial. Lancet 2016;387: 545-557.

21 Portal A, Pernot S, Tougeron D, Arbaud C, Bidault AT, de la Fouchardière $\mathrm{C}$, et al: Nab-paclitaxel plus gemcitabine for metastatic pancreatic adenocarcinoma after Folfirinox failure: an AGEO prospective multicentre cohort. Br J Cancer 2015;113:989995.

22 Waddell N, Pajic M, Patch AM, Chang DK, Kassahn KS, Bailey P, et al: Whole genomes redefine the mutational landscape of pancreatic cancer. Nature 2015;518:495-501.

23 Bailey P, Chang DK, Nones K, Johns AL, Patch AM, Gingras MC, et al: Genomic analyses identify molecular subtypes of pancreatic cancer. Nature 2016;531:47-52. 